\title{
TITLE:
}

\section{CP-Violation in the Renormalizable Theory of Weak Interaction}

AUTHOR(S):

Kobayashi, Makoto; Maskawa, Toshihide

\section{CITATION:}

Kobayashi, Makoto ...[et al]. CP-Violation in the Renormalizable Theory of Weak Interaction. Progress of Theoretical Physics 1973, 49(2): 652-657

ISSUE DATE:

1973-02

URL:

http://hdl.handle.net/2433/66179

RIGHT:

Copyright (c) 1973 Progress of Theoretical Physics 


\title{
CP.Violation in the Renormalizable Theory of Weak Interaction
}

\author{
Makoto KOBAYASHI and Toshihide MASKAWA \\ Department of Physics, Kyoto University, Kyoto
}

(Received September 1, 1972)

\begin{abstract}
In a framework of the renormalizable theory of weak interaction, problems of $C P$-violation are studied. It is concluded that no realistic models of $C P$-violation exist in the quartet scheme without introducing any other new fields. Some possible models of $C P$-violation are also discussed.
\end{abstract}

When we apply the renormalizable theory of weak interaction ${ }^{1)}$ to the hadron system, we have some limitations on the hadron model. It is well known that there exists, in the case of the triplet model, a difficulty of the strangeness changing neutral current and that the quartet model is free from this difficulty. Furthermore, Maki and one of the present authors (T.M.) have shown ${ }^{2)}$ that, in the latter case, the strong interaction must be chiral $S U(4) \times S U(4)$ invariant as precisely as the conservation of the third component of the iso-spin $I_{3}$. In addition to these arguments, for the theory to be realistic, $C P$-violating interactions should be incorporated in a gauge invariant way. This requirement will impose further limitations on the hadron model and the $C P$-violating interaction itself. The purpose of the present paper is to investigate this problem. In the following, it will be shown that in the case of the above-mentioned quartet model, we cannot make a $C P$-violating interaction without introducing any other new fields when we require the following conditions: a) The mass of the fourth member of the quartet, which we will call $\zeta$, is sufficiently large, b) the model should be consistent with our well-established knowledge of the semi-leptonic processes. After that some possible ways of bringing $C P$-violation into the theory will be discussed.

We consider the quartet model with a charge assignment of $Q, Q-1, Q-1$ and $Q$ for $p, n, \lambda$ and $\zeta$, respectively, and we take the same underlying gauge group $S U_{\text {weak }}(2) \times S U(1)$ and the scalar doublet field $\varphi$ as those of Weinberg's original model. ${ }^{1}$ Then, hadronic parts of the Lagrangian can be devided in the following way:

$$
\mathcal{L}_{\text {had }}=\mathcal{L}_{\text {kin }}+\mathcal{L}_{\text {mass }}+\mathcal{L}_{\text {strong }}+\mathcal{L}^{\prime},
$$

where $\mathcal{L}_{\text {kin }}$ is the gauge-invariant kinetic part of the quartet field, $q$, so that it contains interactions with the gauge fields. $\mathcal{L}_{\text {mass }}$ is a generalized mass term of $q$, which includes Yukawa couplings to $\varphi$ since they contribute to the mass of $q$ through the spontaneous breaking of gauge symmetry. $\mathcal{L}_{\text {strong }}$ is a strong-inter- 
action part which conserves $I_{3}$ and therefore chiral $S U(4) \times S U(4)$ invariant. ${ }^{2)}$ We assume $C$ - and $P$-invariance of $\mathcal{L}_{\text {strong. }}$. The last term denotes residual interaction parts if they exist. Since $\mathcal{L}_{\text {mass }}$ includes couplings with $\varphi$, it has possibilities of violating $C P$-conservation. As is known as Higgs phenomena, ${ }^{3}$ three massless components of $\varphi$ can be absorbed into the massive gauge fields and eliminated from the Lagrangian. Even after this has been done, both scalar and pseudoscalar parts remain in $\mathcal{L}_{\text {mass }}$. For the mass term, however, we can eliminate such pseudoscalar parts by applying an appropriate constant gauge transformation on $q$, which does not affect on $\mathcal{L}_{\text {strong }}$ due to gauge invariance.

Now we consider possible ways of assigning the quartet field to representations of the $S U_{\text {weak }}(2)$. Since this. group is commutative with the Lorentz transformation, the left and right components of the quartet field, which are respectively defined as $q_{L} \equiv \frac{1}{2}\left(1+\gamma_{5}\right) q$ and $q_{R} \equiv \frac{1}{2}\left(1-\gamma_{5}\right) q$, do not mix each other under the gauge transformation. Then, each component has three possibilities:

$$
\begin{aligned}
& \text { A) } \mathbf{4}=\mathbf{2}+\mathbf{2}, \\
& \text { B) } \mathbf{4}=\mathbf{2}+\mathbf{1}+\mathbf{1}, \\
& \text { C) } \quad \mathbf{4}=\mathbf{1}+\mathbf{1}+\mathbf{1}+\mathbf{1},
\end{aligned}
$$

where on the r.h.s., $\boldsymbol{n}$ denotes an $n$-dimensional representation of $S U(2)$. The present scheme of charge assignment of the quartet does not permit representations of $n \geq 3$. As a result, we have nine possibilities which we will denote by $(A, A)$, $(A, B), \cdots$, where the former (latter) in the parentheses indicates the transformation properties of the left (right) component. Since all members of the quartet should take part in the weak interaction, and size of the strangeness changing neutral current is bounded experimentally to a very small value, the cases of $(B, C),(C, B)$ and $(C, C)$ should be abandoned. The models of $(B, A)$ and $(C, A)$ are equivalent to those of $(A, B)$ and $(A, C)$, respectively, except relative signs between vector and axial vector parts of the weak current. Since $g_{A} / g_{V}$ ratios are measured only for composite states, this difference of the relative signs would be reduced to a dynamical problem of the composite system. So, we investigate in detail the cases of $(A, A),(A, B),(A, C)$ and $(B, B)$.

i) Case $(A, C)$

This is the most natural choice in the quartet model. Let us denote two ( $S U_{\text {weak }}(2)$ ) doublets and four singlets by $L_{d 1}, L_{d 2}, R_{s 1}^{(p)}, R_{s 2}^{(p)}, R_{s 1}^{(n)}$ and $R_{s 2}^{(n)}$, where superscript $p(n)$ indicates $p$-like ( $n$-like) charge states. In this case, $\mathcal{L}_{\text {mass }}$ takes, in general, the following form:

$$
\begin{gathered}
\mathcal{L}_{\text {mass }}=\sum_{i, j=1,2}\left[M_{i j}^{(n)} \bar{L}_{d i} \varphi R_{s j}^{(n)}+M_{i j}^{(p)} \bar{L}_{d i} \varepsilon \varphi^{*} R_{s j}^{(p)}\right]+\text { h.c. }, \\
\varphi^{*} \equiv\left(\begin{array}{c}
\varphi^{-} \\
\bar{\varphi}^{0}
\end{array}\right), \quad \varepsilon \equiv\left(\begin{array}{cc}
0 & 1 \\
-1 & 0
\end{array}\right),
\end{gathered}
$$


where $M_{i j}^{(n)}$ and $M_{i j}^{(p)}$ are arbitrary complex numbers. We can eliminate three Goldstone modes $\phi_{i}$ by putting

$$
\varphi=e^{i \phi_{i \tau}}\left(\begin{array}{c}
0 \\
\lambda+\sigma
\end{array}\right)
$$

where $\lambda$ is a vacuum expectation value of $\varphi^{0}$ and $\sigma$ is a massive scalar field. Thereafter, performing a diagonalization of the remaining mass term, we obtain

$$
\begin{aligned}
\mathcal{L}_{\text {mass }} & =\bar{q} m q\left(1+\frac{\sigma}{\lambda}\right), \\
m & \equiv\left(\begin{array}{cccc}
m_{p} & 0 & 0 & 0 \\
0 & m_{n} & 0 & 0 \\
0 & 0 & m_{\zeta} & 0 \\
0 & 0 & 0 & m_{\lambda}
\end{array}\right), \quad q \equiv\left(\begin{array}{c}
p \\
n \\
\zeta \\
\lambda
\end{array}\right) .
\end{aligned}
$$

Then, the interaction with the gauge field in $\mathcal{L}_{\text {kin }}$ is expressed as

$$
\sum_{j=1}^{3} A_{\mu}^{j} i \bar{q} \Lambda_{j} \gamma_{\mu} \frac{1+\gamma_{5}}{2} q .
$$

Here, $A_{j}$ is the representation matrix of $S U_{\text {weak }}(2)$ for this case and explicitly given by

$$
\Lambda_{+}=\frac{\Lambda_{1}+i \Lambda_{2}}{2}=K\left(\begin{array}{cc}
0 & U \\
0 & 0
\end{array}\right) K^{-1}, \quad \Lambda_{3}=\left(\begin{array}{cccc}
1 & 0 & 0 & 0 \\
0 & 1 & 0 & 0 \\
0 & 0 & -1 & 0 \\
0 & 0 & 0 & -1
\end{array}\right), \quad K \equiv\left(\begin{array}{llll}
1 & 0 & 0 & 0 \\
0 & 0 & 1 & 0 \\
0 & 1 & 0 & 0 \\
0 & 0 & 0 & 1
\end{array}\right)
$$

where $U$ is a $2 \times 2$ unitary matrix. Here and hereafter we neglect the gauge field corresponding to $U(1)$ which is irrelevant to our discussion. With an appropriate phase convention of the quartet field we can take $U$ as

$$
U=\left(\begin{array}{cc}
\cos \theta & \sin \theta \\
-\sin \theta & \cos \theta
\end{array}\right) \text {. }
$$

Therefore, if $\mathcal{L}^{\prime}=0$, no $C P$-violations occur in this case. It should be noted, however, that this argument does not hold when we introduce one more fermion doublet with the same charge assignment. This is because all phases of elements of a $3 \times 3$ unitary matrix cannot be absorbed into the phase convention of six fields. This possibility of $C P$-violation will be discussed later on.

ii) Case $(A, B)$

This is a rather delicate case. We denote two left doublets, one right doublet and two singlets by $L_{d 1}, L_{d 2}, R_{d}, R_{s}{ }^{(p)}$ and $R_{s}{ }^{(n)}$, respectively. The general form 
of $\mathcal{L}_{\text {mass }}$ is given by

$$
\mathcal{L}_{\text {mass }}=\sum_{i=1,2}\left[m_{i} \bar{L}_{d i} R_{d}+M_{i}^{(n)} \bar{L}_{d i} \varphi R_{s}^{(n)}+M_{i}^{(p)} \bar{L}_{d i} \varepsilon \varphi^{*} R_{s}^{(p)}\right]+\text { h.c. },
$$

where $m_{i}, M_{i}^{(n)}$ and $M_{i}{ }^{(p)}$ are arbitrary complex numbers. After diagonalization of mass terms (in this case, the $C P$-odd part of coupling with $\sigma$ does not disappear in general) each multiplet can be expressed as follows:

$$
\begin{array}{ll}
L_{a 1}=\frac{1+\gamma_{5}}{2}\left(\begin{array}{c}
p \\
\cos \theta e^{i \alpha} n+\sin \theta e^{i \beta} \lambda
\end{array}\right), & L_{d 2}=\frac{1+\gamma_{5}}{2}\left(\begin{array}{c}
e^{i \gamma} \zeta \\
-\sin \theta e^{i \alpha} n+\cos \theta e^{i \beta} \lambda
\end{array}\right), \\
R_{d}=\frac{1-\gamma_{5}}{2}\left(\begin{array}{cl}
\sin \xi \cdot p+\cos \xi \cdot \zeta \\
\sin \eta \cdot n+\cos \eta \cdot \lambda
\end{array}\right), & R_{s}^{(p)}=\frac{1-\gamma_{5}}{2}(\cos \xi \cdot p-\sin \xi \cdot \zeta), \\
& R_{s}^{\left({ }^{(n)}\right.}=\frac{1-\gamma_{5}}{2}(\cos \eta \cdot n-\sin \eta \cdot \lambda),
\end{array}
$$

where phase factors $\alpha, \beta$ and $\gamma$ satisfy two relations with the masses of the quartet:

$$
\begin{aligned}
& e^{i r} m_{\zeta} \sin \theta \cos \xi=m_{p} \cos \theta \sin \xi-e^{i \alpha} m_{n} \sin \eta, \\
& e^{i r} m_{\zeta} \cos \theta \cos \xi=-m_{p} \sin \theta \cos \xi+e^{i \beta} m_{\lambda} \cos \eta .
\end{aligned}
$$

Owing to the presence of phase factors, there exists a possibility of $C P$-violation also through the weak current. However, the strangeness changing neutral current is proportional to $\sin \eta \cos \eta$ and its experimental upper bound is roughly

$$
\sin \eta \cos \eta<10^{-2 \sim-3} \text {. }
$$

Thus, making an approximation of $\sin \eta \sim 0$ (the other choice $\cos \eta \sim 0$ is less critical) we obtain from Eq. (8)

$$
\begin{aligned}
& m_{\zeta} / m_{p} \sim \cot \theta \cdot \tan \xi, \\
& m_{\lambda} / m_{n} \sim \sin \xi / \sin \theta .
\end{aligned}
$$

We have no low-lying particle with a quantum number corresponding to $\zeta$, so that $m_{\zeta}$, which is a measure of chiral $S U(4) \times S U(4)$ breaking, should be sufficiently large compared to the masses of the other members. However, the present experimental results on the $g_{A} / g_{V}$ ratios of the octet baryon $\beta$-decay would not permit $\sin \xi>\sin \theta$. Thus, it seems difficult to reconcile the hierarchy of chiral symmetry breaking with the experimental knowledge of the semileptonic processes.

iii) Case $(B, B)$

As a previous one, in this case also, occurrence of $C P$-violation is possible, but in order to suppress $|\Delta S|=1$ neutral currents, coefficients of the axial-vector part of $\Delta S=0$ and $|\Delta S|=1$ weak currents must take signs oppossite to each other. This contradicts again the experiments on the baryon $\beta$-decay. 
iv) Case $(A, A)$

In a similar way, we can show that no $C P$-violation occurs in this case as far as $\mathcal{L}^{\prime}=0$. Furthermore this model would reduce to an exactly $U(4)$ symmetric one.

Summarizing the above results, we have no realistic models in the quartet scheme as far as $\mathcal{L}^{\prime}=0$. Now we consider some examples of $C P$-violation through $\mathcal{L}^{\prime}$. Hereafter we will consider only the case of $(A, C)$. The first one is to introduce another scalar doublet field $\psi$. Then, we may consider an interaction with this new field

$$
\begin{aligned}
& \mathcal{L}^{\prime}=\bar{q} \psi \boldsymbol{C} \frac{1-\gamma_{5}}{2} q+\text { h.c. }, \\
& \psi \equiv\left(\begin{array}{cccc}
\bar{\phi}^{0} & \phi^{+} & 0 & 0 \\
-\psi^{-} & \psi^{0} & 0 & 0 \\
0 & 0 & \bar{\psi}^{0} & \phi^{+} \\
0 & 0 & -\psi^{-} & \phi^{0}
\end{array}\right), \quad \boldsymbol{C} \equiv\left(\begin{array}{cccc}
c_{11} & 0 & c_{12} & 0 \\
0 & d_{11} & 0 & d_{12} \\
c_{21} & 0 & c_{22} & 0 \\
0 & d_{21} & 0 & d_{22}
\end{array}\right) \text {, }
\end{aligned}
$$

where $c_{i j}$ and $d_{i j}$ are arbitrary complex numbers. Since we have already made use of the gauge transformation to get rid of the CP-odd part from the quartet mass term, there remains no such arbitrariness. Furthermore, we note that an arbitrariness of the phase of $\psi$ cannot absorb all the phases of $c_{i j}$ and $d_{i j}$. So, this interaction can cause a $C P$-violation.

Another one is a possibility associated with the strong interaction. Let us consider a scalar (pseudoscalar) field $S$ which mediates the strong interaction. For the interaction to be renormalizable and $S U_{\text {weak }}(2)$ invariant, it must belong to a $\left(4,4^{*}\right)+\left(4^{*}, 4\right)$ representation of chiral $S U(4) \times S U(4)$ and interact with $q$ through scalar and pseudoscalar couplings. It also interacts with $\varphi$ and possible renormalizable forms are given as follows:

$$
\begin{aligned}
& \operatorname{tr}\left\{G_{0} S^{+} \boldsymbol{\varphi}\right\}+\text { h.c., } \\
& \operatorname{tr}\left\{G_{1} S^{+} \varphi G_{2} \varphi^{+} S\right\}+\text { h.c. }, \\
& \operatorname{tr}\left\{G_{1}{ }^{\prime} S^{+} \varphi G_{2}{ }^{\prime} S^{+} \varphi\right\}+\text { h.c. },
\end{aligned}
$$

with

$$
\varphi \equiv\left(\begin{array}{cccc}
\bar{\varphi}^{0} & \varphi^{+} & 0 & 0 \\
-\varphi^{-} & \varphi^{0} & 0 & 0 \\
0 & 0 & \bar{\varphi}^{0} & \varphi^{+} \\
0 & 0 & -\varphi^{-} & \varphi^{0}
\end{array}\right),
$$

where $G_{i}$ is a $4 \times 4$ complex matrix and we have used a $4 \times 4$ matrix representation for $S$. It is easy to see that these interaction terms can violate $C P$-conservation. 
Next we consider a 6-plet model, another interesting model of $C P$-violation. Suppose that 6-plet with charges $(Q, Q, Q, Q-1, Q-1, Q-1)$ is decomposed into $S U_{\text {weak }}$ (2) multiplets as $2+2+2$ and $1+1+1+1+1+1$ for left and right components, respectively. Just as the case of $(A, C)$, we have a similar expression for the charged weak current with a $3 \times 3$ instead of $2 \times 2$ unitary matrix in Eq. (5). As was pointed out, in this case we cannot absorb all phases of matrix elements into the phase convention and can take, for example, the following expression:

$\left(\begin{array}{lll}\cos \theta_{1} & -\sin \theta_{1} \cos \theta_{3} & -\sin \theta_{1} \sin \theta_{3} \\ \sin \theta_{1} \cos \theta_{2} & \cos \theta_{1} \cos \theta_{2} \cos \theta_{3}-\sin \theta_{2} \sin \theta_{3} e^{i \delta} & \cos \theta_{1} \cos \theta_{2} \sin \theta_{3}+\sin \theta_{2} \cos \theta_{3} e^{i \delta} \\ \sin \theta_{1} \sin \theta_{2} & \cos \theta_{1} \sin \theta_{2} \cos \theta_{3}+\cos \theta_{2} \sin \theta_{3} e^{i \delta} & \cos \theta_{1} \sin \theta_{2} \sin \theta_{3}-\cos \theta_{2} \sin \theta_{3} e^{i \delta}\end{array}\right)$.

Then, we have $C P$-violating effects through the interference among these different current components. An interesting feature of this model is that the $C P$-violating effects of lowest order appear only in $\Delta S \neq 0$ non-leptonic processes and in the semi-leptonic decay of neutral strange mesons (we are not concerned with higher states with the new quantum number) and not in the other semi-leptonic, $\Delta S=0$ non-leptonic and pure-leptonic processes.

So far we have considered only the straightforward extensions of the original Weinberg's model. However, other schemes of underlying gauge groups and/or scalar fields are possible. Georgi and Glashow's model ${ }^{4)}$ is one of them. We can easily see that $C P$-violation is incorporated into their model without introducing any other fields than (many) new fields which they have introduced already.

\section{References}

1) S. Weinberg, Phys. Rev. Letters 19 (1967), 1264; 27 (1971), 1688.

2) Z. Maki and T. Maskawa, RIFP-146 (preprint), April 1972.

3) P. W. Higgs, Phys. Letters 12 (1964), 132; 13 (1964), 508.

G. S. Guralnik, C. R. Hagen and T. W. Kibble, Phys. Rev. Letters 13 (1964), 585.

4) H. Georgi and S. L. Glashow, Phys. Rev. Letters 28 (1972), 1494. 